\title{
Frequency of Reporting and the Quality of Randomized Controlled Trials Published in the Nigerian Journal of Ophthalmology
}

\author{
${ }^{1} \mathrm{H}$ Ejere мввS, MSc, FWACS, ${ }^{2} \mathrm{MB}$ Alhassan мввS, FWACS \\ ${ }^{1}$ Inpatient Medicine Specialists, Phoebe Putney Memorial Hospital, 425 W. Third Avenue, Albany USA \\ ${ }^{2}$ Department of Clinical Ophthalmology, National Eye Centre, PMB 2267, Kaduna
}

\section{SUMMARY}

Aim: To determine the frequency of reporting and the methodological quality of randomized controlled trials in the Nigerian Journal of Ophthalmology (NJO) from 1993 - 2001.

Materials and methods: Back issues of NJO published from 1993 to 2001 were searched for reports of randomized controlled trials. The quality of identified trials was assessed using standard Cochrane methods.

Results: One out of 104 articles published in the 9 volumes of NJO from 1993 - 2001 can be described as a randomized controlled trial. Complete information regarding the method of allocation concealment, masking of providers and recipients of care, and masking of outcome assessors was not provided. It was not clear whether all patients were followed-up for 6 months or 1 year, or whether there was a differential loss to follow-up between the study groups.

Conclusions: Only one randomized controlled trial was published in the Nigerian Journal of Ophthalmology between 1993-2001. Assessment of the methodological quality of the reported trial was hampered by lack of complete information on parameters used to assess trial quality in this study.

Key words: clinical trials, reporting, methodological quality, Nigerian Journal of Ophthalmology

\section{INTRODUCTION}

A clinical trial is defined as a prospective study comparing the effect and value of intervention(s) against a control in human subjects. ${ }^{1}$ Randomized controlled trials (RCTs) are generally regarded as the gold standard in scientific research when considering questions of therapeutic efficacy. While evidence from case control studies and cohort studies can be used to answer questions of efficacy, confounding factors are usually a major cause of concern, especially if they are not known or have not been measured. The process of randomization in RCTs, if properly done, helps to control for the effect of puzzling variables. It is, therefore, believed that RCTs are likely to provide more reliable information than other sources of evidence on the differential effects of alternative forms of healthcare interventions. ${ }^{2}$

A poorly designed and conducted RCT, however, may provide misleading information on the effects of a health care intervention. Therefore, the methodological quality of RCTs is important in determining the validity or reliability of trial results. Several scales and checklists have been used to assess the quality of trials, ${ }^{3}$ but these scales and checklists have not been shown to provide consistent and reliable assessment of validity. ${ }^{4}$ In this study, we adopted a simple approach, using parameters which address important substantive threats to study validity. ${ }^{5}$ Concealment of allocation helps to minimize selection bias (systematic differences in comparison groups), while masking of patients and providers of treatment helps to reduce performance bias (systematic differences in care provided apart from the intervention being evaluated). Masking of outcome assessors helps to reduce detection bias (systematic differences in outcome assessment). A complete follow-up or good account of losses to follow-up helps to reduce attrition bias (systematic differences in withdrawals from the trial).

The practice of ophthalmology is evolving rapidly due to the introduction of new interventions in the management of ophthalmic diseases. A good example is the shift from intracapsular cataract extraction to extracapsular cataract extraction and more recently to small incision techniques with phacoemulsification. Similarly, antimetabolites have been introduced in some centres in the surgical management of glaucoma when indicated.

Ophthalmologists are often faced with the need to choose between alternative forms of health care interventions in the management of patients. Although evidence from trials of interventions conducted elsewhere and in different populations can inform these choices, there is, however, usually some uncertainty regarding the applicability of such interventions in patients drawn from African populations. There is, therefore, a need to conduct trials among African patients to test the efficacy and safety of some of these new interventions, before they are generally adopted. The Nigerian Journal of Ophthalmology (NJO) is the official organ of the Ophthalmological Society of Nigeria and a source of information regarding ophthalmic research

${ }^{*}$ Correspondence: Dr. H Ejere, Inpatient Medicine Specialists, Phoebe Putney Memorial Hospital, 425 W. Third Avenue, Albany, USA • email: cpegbe@weltekng.com 
conducted by ophthalmologists practising in Nigeria. Ophthalmologists practising in Nigeria may therefore rely on NJO as a source of information with respect to clinical trials conducted in Nigeria. Therefore, a survey of NJO was carried out from 1993-2001 to determine the frequency of reporting and the methodological quality of randomized controlled trials published therein.

\section{METHODS}

Back issues of NJO from 1993 to 2001 were reviewed in order to identify randomized controlled trials (RCTs); article titles, summaries/abstracts, and methodologies of published articles were manually searched using the following search terms: clinical trial; controlled trial; randomization; masking/blinding, or placebo. Methodological quality was assessed according to methods set out in section six of the Cochrane Reviewer's Handbook. ${ }^{5}$ The following parameters were considered: concealment of allocation, masking of providers and recipients of care, masking of outcome assessors, and adequacy or comparability of follow-up rates in treatment and control groups.

Concealment of allocation was considered adequate if allocation was by centralized randomization either by a central office or pharmacy; the sequential administration of pre-numbered or coded containers to enrolled participants, serially numbered sealed opaque envelopes, or on-site computer systems provided the computer file containing the assignment is locked. Allocation based on alternation, case records numbers, dates of birth, or day of the week was considered inadequate, as they are likely to lead to selection bias.

\section{RESULTS}

Frequency of reporting of randomized controlled trials

A total of 104 articles were published in 9 volumes of NJO from 1993 to 2001 (table 1). Only one of these articles (0.96\%) can be described as a randomized controlled trial. ${ }^{6}$

Table 1. Total number of published articles in NJO (1993 to 2001)

\begin{tabular}{ccccc}
\hline Year & Vol & Issue & Articles & RCTs \\
\hline 1993 & 2 & 1 & 18 & 0 \\
1994 & 2 & 2 & 11 & 0 \\
1995 & 3 & 2 & 12 & 0 \\
1996 & 4 & 1 & 12 & 0 \\
1997 & 5 & 1 & 15 & 0 \\
1998 & 6 & 1 & 8 & 0 \\
1999 & 7 & 1 & 10 & 1 \\
2000 & 8 & 1 & 7 & 0 \\
2001 & 9 & 1 & 11 & 0 \\
\hline TOTAL & & & $\mathbf{1 0 4}$ & $\mathbf{1}$ \\
\hline
\end{tabular}

Participants in this trial comprised 32 patients diagnosed as having pterygium (fleshy and vascular type). Patients were randomized to receive bare sclera plus 5-fluorouracil $\{50 \mathrm{mg} / \mathrm{ml}\}$ (20 patients and 28 eyes) or bare sclera technique alone (12 patients and 16 eyes). Outcomes were recurrence rate and complications. The intended period of follow-up was not stated at the outset.

\section{Methodological quality of trial}

Concealment of allocation: Method of allocation of patients to treatment groups was not stated.

Masking of providers and recipients: Not stated

Masking of outcome assessors: Not stated

Completeness of follow up: Not clear whether there were any losses to follow up and whether all patients were followed up for 6 months or one year.

\section{DISCUSSION}

The finding of only one randomized controlled trial (RCT) among 104 published articles in 9 issues of the NJO from 1993 - 2001 seems to suggest the low priority given to this type of study design by ophthalmologists involved in research in Nigeria. For many obvious reasons, we cannot directly compare the NJO with the British Journal of Ophthalmology (BJO), but a quick electronic search of Medline (Pubmed) showed that about 15\% (98 out of 639) of articles published in the BJO from 1993-2001 were indexed as clinical trials.

The present study cannot answer the question as to how many RCTs relevant to ophthalmology are actually conducted within Nigeria, or how many of these are published in other journals outside Nigeria. However, a qualitative study is underway to assess the knowledge and attitude to RCTs among Nigerian ophthalmologists. This is important given the role of RCTs in providing reliable evidence to inform and direct practice.

Assessment of the quality of the only identified trial was difficult as the report of this trial did not include details of how randomization was done, whether allocation of patients to treatment or control groups was concealed or not, or whether providers and recipients of treatment were masked. Information on whether outcome assessment i.e. recurrence rate and complications was masked or not was not stated. Although it was stated that follow-up was between 6-12 months, it is unclear whether all the patients were followedup within that period.

It is not certain if the lack of reporting of quality parameters in the identified trial was due to the fact that they were not considered in the design of the trial or whether they were considered but not reported, because of journal restrictions on the number of words or pages per article.

Good reporting of a trial facilitate the understand of what was done in the trial, how it was done and how the authors attempted to prevent bias. A standard for reporting of trials has been developed and published, ${ }^{7}$ and is known 
as the Consolidated Standards of Reporting Trials (CONSORT). The CONSORT statement was designed to ensure adequate reporting of randomised controlled trials by listing over 20 items, including a flow chart describing the participants' progress through the trial that should be included in a report. The Nigerian Journal of Ophthalmology should insist that all randomized trial authors should follow CONSORT statement to ensure that their R CT trials provide reliable information.

\section{REFERENCES}

1. Friedman LM, Furberg CD, DeMets D. Fundamentals of Clinical Trials. PSG Publishing Company, .Littleton, Massachusetts, 1985, p. 2.

2. Kunz R, Oxman AD. The unpredictability paradox: review of empirical comparisons of randomised and non-randomised clinical trials. BMJ 1998; 317: 1185-90.

3. Moher D, Jadad A, Nichol G, Penman M, Tugwell T, Walsh S. Assessing the quality of randomized controlled trials: an annotated bibliography of scales and checklists. Controlled Clin Trials 1995; 16: 62-73.

4. Jüni $\mathrm{P}$, Witschi A, Bloch R, Egger M. The hazards of scoring the quality of clinical trial for meta-analysis. JAMA 1999; 282: 1054-60.

5. Clarke M, Oxman AD, editors. Assessment of study quality. Cochrane Reviewers' Handbook 4.1 [updated June 2000]; Section 6. In: Review Manager (RevMan) [Computer program]. Version 4.1. Oxford, England: The Cochrane Collaboration, 2000

6 Ukponmwan CU, Waziri-Erameh MJM. Effect of intraoperative application of 5-fluorouracil on recurrence rate of pterygium Benin City, Nigeria. Nigerian Journal of Ophthalmology 1999; 7(1): 10-13.

7. Begg C, Cho M, Eastwood S, Horton R, Moher D, Olkin I, et al. Improving the quality of reporting of randomized controlled trials. The CONSORT statement. Journal of the American Medical Association 1996; 276(8): 637-9. 